\title{
In Situ Fiber Optic Dissolution Analysis
}

Paul K. Aldridge and Leonard J. Kostek

Analytical Research and Development Pfizer Central Research, Groton, CT
$\mathrm{P}$

fizer Inc. has configured an automated dissolution system utilizing a fiber-optic probe for ultraviolet spectrophotometric in situ end ans a Hewlett-Packard 8452A spectrophotometer. The probe is moved from vessel to vessel for sampling purposes by a Zymark System V robot arm. A paper has been published in the Journal of Pharmaceutical Sciences ${ }^{1}$ which describes this automated dissolution method and outlines the software configuration. This system was built with standard, off-the-shelf hardware and software. The only custom pieces were derived from an in-house Matlab software program to handle all the communication and data transfer and a user-generated Zymate program $^{3}$ to operate the Zymark robot.

The system can use either a $10 \mathrm{~mm}, 5$ $\mathrm{mm}$ or $2 \mathrm{~mm}$ equivalent path fiber-optic probe connected to the spectrophotometer. The robot moves the probe from vessel to vessel for sampling the dissolution media at 5 minute intervals. Each sampling point is completed in 2 seconds with the acquisition of 20 spectra from 190 to $820 \mathrm{~nm}$ using the program DeLight. ${ }^{4}$ The scans are averaged and sent to a $\mathrm{Matlab}^{2}$ program where a second derivative is performed to eliminate background interference. This mathematical background correction eliminates the need for filtration of samples. Avoiding the use of filters saves time in the method development process, plus circumvents future problems with drug holdup on the filter or availability of suitable filters. The data points are plotted in real time on a graphic display screen for each vessel as indicated in Figure 1. Final dissolution results are summarized as shown in Figure 2 with error bars indicating high, low and average results for the total run. Specification limits for time and percent dissolved have been included in the display for an immediate indica- tion of sample performance with respect to specification limits.

This automated dissolution system is capable of performing 12 unassisted dissolution tests with one setup and up to 18 runs in one day. Vessels are automatically filled with dissolution medium and the medium temperature is equilibrated to $37^{\circ} \mathrm{C} \pm 0.5^{\circ} \mathrm{C}$. Dosage forms are introduced to the vessels, sampling occurs at the specified time intervals and the vessels are automatically cleaned at the end of each run. The ability to gather additional data points, up to twelve data points per hour, as compared to previous dissolution methods provides a more complete dissolution profile. 


\section{Real-Time Dissolution Results $10 \mathrm{mg}$ Tablets}
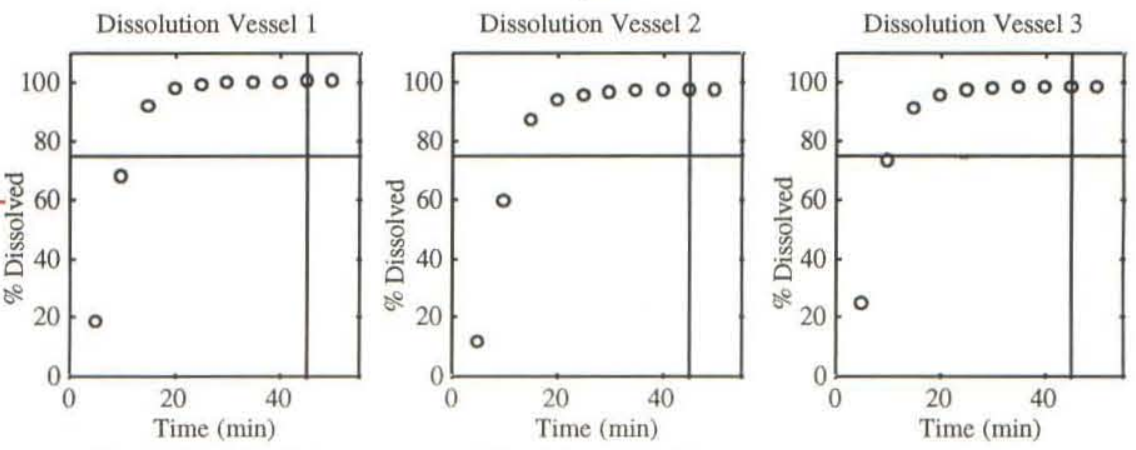

Since data are collected in real time, this unit can be a valuable tool in formulation development as well as routine testing. This system provides high throughput with efficient sampling, no sample loss for analysis and added safety due to less employee exposure to high potency compounds.

' Aldridge, P.K., et. al. J. Pharm. Sci., 84(8), p. 909-914, August, 1995.

${ }^{2}$ Zymark Corp., Hopkinton, MA.

${ }^{3}$ Matlab (Matlab version 4.2c.1, The Mathworks Inc., Natick, MA).

4 DeLight, (version 1.2, D Squared Development Inc., LaGrande, OR).
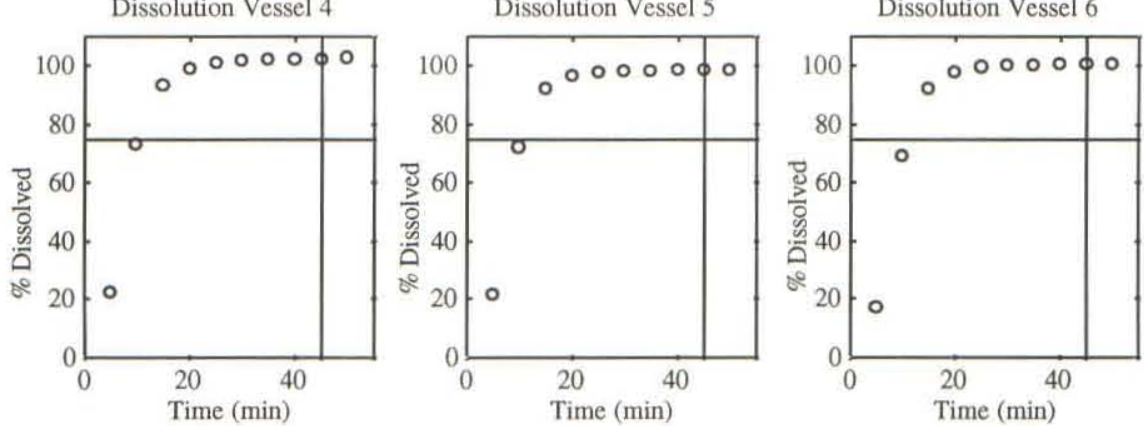

Figure 1. Dissolution Profiles from a Six (6) Vessel Dissolution Run. Results are Updated in Real-Time as the Experiment Proceeds.

\section{Dissolution Results Summary \\ $10 \mathrm{mg}$ Tablets}

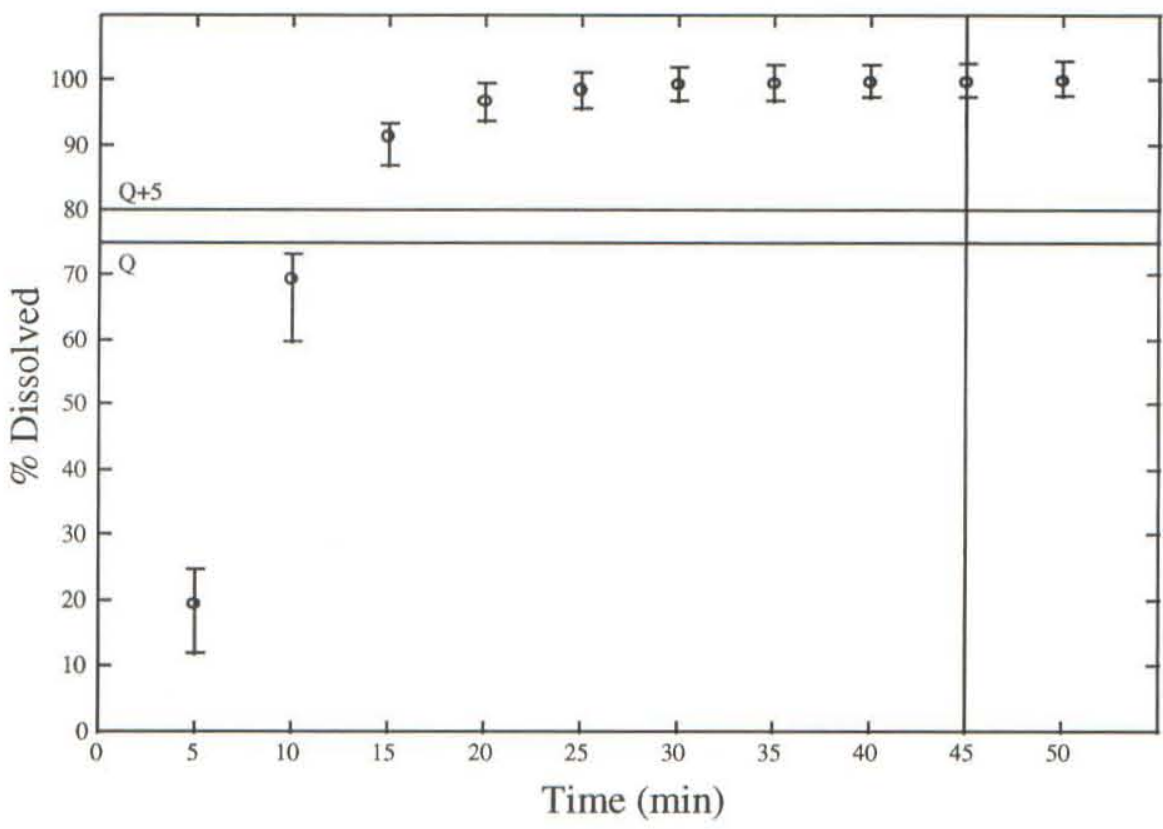

Figure 2. Summary Dissolution Profile: Error Bars Represent Max and Min Values 